\title{
POWER INTERFACES AND STORAGE SELECTION FOR AN ULTRAFAST EV CHARGING STATION
}

\author{
H. Hõimoja, M. Vasiladiotis, A. Rufer \\ Swiss Federal Institute of Technology Lausanne, Switzerland, EPFL-LEI, Station 11, 1015 Lausanne, \\ hardi.hoimoja@epfl.ch
}

Keywords: Electric Vehicle, Energy Management, Energy Storage, Cascaded H-bridge.

\begin{abstract}
The paper is a summary of the research conducted so far on the ultrafast charging issues of electric vehicles with the main emphasis on the infrastructure. In order to estimate the load curve and peaks resulting from high charging rate, simulations are carried out initially to determine energy and power ratings. Energy storage options for filtering out the perspective peaks are discussed with applicable power interfaces between the grid, the vehicle and the storage buffer. Finally, an ultrafast charging architecture based on cascaded $\mathrm{H}$-bridge converters is proposed.
\end{abstract}

\section{Introduction}

The electric vehicles (EV) are being continuously promoted by the manufacturers and the governments willing to excel in their environmental awareness. Unfortunately, their good will is in reality limited by existing possibilities concerning the autonomy and charging availability of such cars. The commercialised EVs have datasheet autonomy around $160 \mathrm{~km}$ with charging times from $6 \mathrm{~h} \ldots 8 \mathrm{~h}$ in domestic conditions down to $20 \mathrm{~min}$ if state-of-the-art public quick chargers are employed [1]. The actual problem may be formulated by two distinctive EV-related terms of autonomy and autonomy flowrate:

1. Autonomy is the average distance an EV is able to cover with the maximally recharged battery in given conditions (capacity, charging current, initial state of charge etc.).

2. Autonomy flowrate is the driving distance augmentation in time, expressed in $\mathrm{km} / \mathrm{min}$ or $\mathrm{km} / \mathrm{h}$.

These two values determine the quantity and duration of intermediate recharges during a trip and as follows, the total travel time and average speed. For explaining the actual situation, a fuel-efficient diesel family car and a generic EV are compared in Table 1 . Whereas the fuel tank capacity is independent of the tanking speed, the available capacity of an electrochemical battery is de-rated at high charging rates, additional influence posed by the ambient temperature. The tanking speed (resp. charging power) is directly related to autonomy flowrate; on the other side, the autonomy of an EV decreases while increasing the charging power. Thus a trade- off between autonomy flowrate and autonomy itself is inevitable. However, bearing in mind that an average driver rarely drives hundreds of kilometres non-stop, the autonomy could be de-ranked if ultrafast charging infrastructure coverage is sufficient to provide energy if necessary, not only if available as today [2].

\begin{tabular}{|l|l|l|}
\hline Parameter & Diesel car & Electric vehicle \\
\hline Consumption & $51 / 100 \mathrm{~km}$ & $15 \mathrm{~kW} \cdot \mathrm{h} / 100 \mathrm{~km}$ \\
\hline Tank capacity & 451 & $24 \mathrm{~kW} \cdot \mathrm{h}$ \\
\hline Autonomy & $900 \mathrm{~km}$ & $120 \mathrm{~km}$ \\
\hline Tanking speed & $351 / \mathrm{min}$ & $50 \mathrm{~kW}$ \\
\hline Autonomy flowrate & $700 \mathrm{~km} / \mathrm{min}$ & $4 \mathrm{~km} / \mathrm{min}$ \\
\hline
\end{tabular}

Table 1: Autonomy and autonomy flowrate comparisons

\section{Energy and power requirements}

To determine the design parameters for an ultrafast EV charging station (UFCS), several data must be known or predefined:

1) objective charging time;

2) useful battery capacity at objective charging time;

3) initial state of charge (SoC) at the start of charging;

4) efficiencies of energy conversion stages;

5) UFCS utilisation.

\subsection{Battery charging process}

During charging, the system charger-battery draws not only useful power from the grid, which is accumulated as traction energy for later use, but also additional components to compensate the losses inside the battery $P_{l o s s, b}$ and the charging converter $P_{\text {loss,conv }}$ (Fig. 1) As the battery has resistive characteristics caused by the sum of internal and contact resistances of individual cells $\sum R_{\text {cell }}$, its losses augment with charging current $i_{c h}$ squared. The power $P_{g}$ posed by recharging battery to the grid can be expressed as

$$
P_{g}=\frac{1}{\eta_{\text {conv }}} \cdot i_{c h} \cdot\left[u_{o c}(\operatorname{SoC})+i_{c h} \cdot \sum R_{\text {cell }}\right]
$$

The open circuit voltage $u_{o c}$ of a battery can be considered analogous to counter-emf, increasing with $S o C$ in a non-linear manner. With nearly constant charger converter efficiency $\eta_{c o n v}$, the grid power must be increased during recharging to keep the constant charging current. 


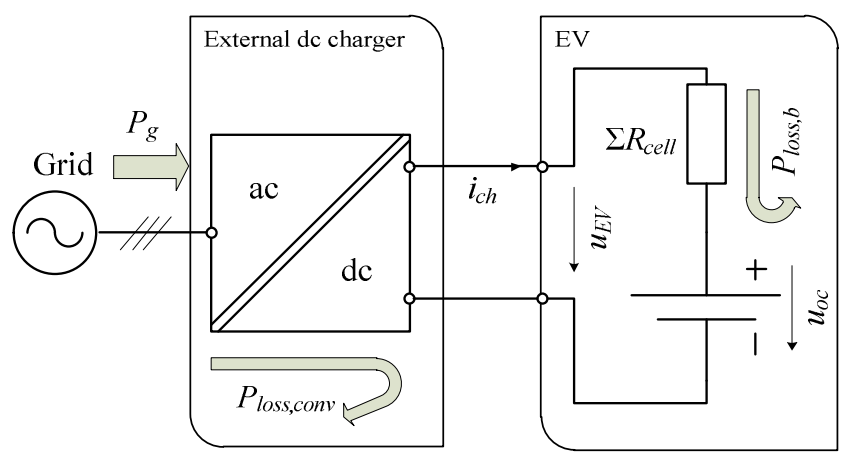

Fig. 1: Energy flow from grid to battery

\subsection{Considerations on utilisation}

While forecasting the load profile of an UFCS, several assumptions have been made:

1. EV rated battery capacities vary from one vehicle model to another. Moreover, the capacity is prone to ageing and temperature; therefore in simulations the capacities are subjected to left-truncated normal distribution, as shown in Fig. 2.

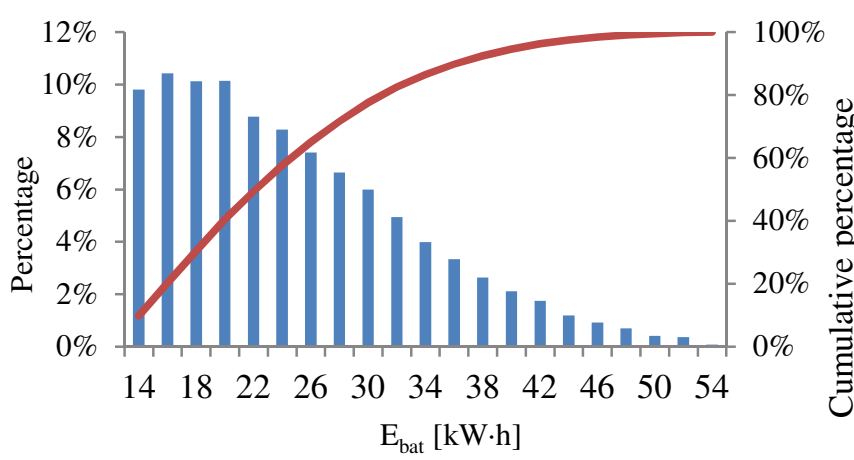

Fig. 2: EV battery capacity $E_{b a t}$ histogram

2. The vehicles arriving at an UFCS have varying initial state of charge, representing the remaining energy inside the battery. The values are subjected to normal distribution as shown in Fig. 3.

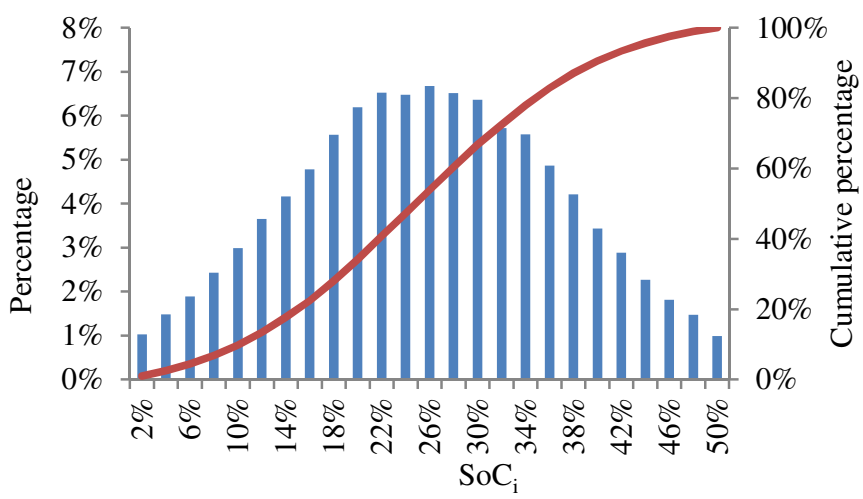

Fig. 3: EV battery initial state of charge $S o C_{i}$ histogram

At the given number of vehicles being charged during $24 \mathrm{~h}$, their arrival intervals at an UFCS are uneven, depending on the traffic density in the given hour (Fig. 4).

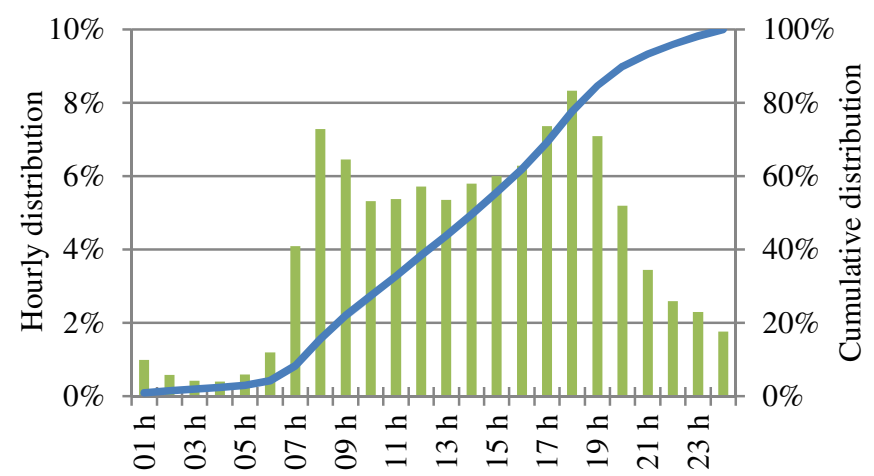

Hourly distribution $\longrightarrow$ Cumulative distribution

Fig. 4: Typical traffic density distribution in Switzerland

\subsection{UFCS load curve generation procedure}

The presumed load curve is deducted from the objective number of EV to be charged during a day. For next, following steps are performed:

1. Hourly distribution of vehicles according to Fig. 4.

2. Assignment of arrival times within the specified hour. A vehicle can arrive at any minute of the given hour, so the minute value is generated randomly.

3. Assignment of the initial state-of-charge $S o C_{i}$ and rated capacity $E_{b a t}$ to each EV.

4. Generating charging curve for each EV based on the objective charging time $t_{c h}$.

5. Superimposing charging curves to achieve sum values.

The EV battery rated voltage $U_{E V, n}=330 \mathrm{~V}$ has been considered independent of the vehicle, this value being characteristic to small EVs such as C-Zero, iMiEV and $i O n$. The $S o C$ for the effective energy exchange range of an $\mathrm{EV}$ battery is taken between $100 \% \ldots 0 \%$ and the resistance is considered reciprocal to its capacity. The base value $\Sigma R_{\text {cell }}(16 \mathrm{~kW} \cdot \mathrm{h})=70.4 \mathrm{~m} \Omega$ is taken from a datasheet [3].

$$
\Sigma R_{\text {cell }}\left(E_{\text {bat }}\right)=\Sigma R_{\text {cell }}(16 \mathrm{~kW} \cdot \mathrm{h}) \cdot \frac{16 \mathrm{~kW} \cdot \mathrm{h}}{E_{\text {bat }}}
$$

The charging current $i_{c h}$ is determined by the battery capacity and objective charging time $t_{c h}$ and the open circuit voltage $u_{o c}$ is considered to have linear relationship to $\mathrm{SoC}$ with $U_{0}=0.8 \cdot U_{b a t, N}$

$$
u_{o c}(\operatorname{SoC})=(0.8+0.2 \cdot \operatorname{SoC}) \cdot u_{E V, n}
$$

The $S o C$ estimation itself is based on the coulomb count:

$$
\operatorname{SoC}(t)=\operatorname{SoC}_{i}+\frac{\Delta t}{Q_{b a t}} \cdot \sum_{\tau=0}^{t} i_{c h, \tau},
$$

where $Q_{b a t}=E_{b a t} / U_{E V, n}$ is the so-called coulomb capacity of a battery and $\Delta t$ numeric integration timestep. The charging process is terminated when $S o C \approx S o C_{\max }$.

The battery terminal voltage is a sum of $u_{o c}$ and the voltage drop across the internal resistance: 


$$
u_{E V}(t)=u_{o c}(S o C)+i_{c h} \cdot \Sigma R_{c e l l}
$$

The charging power $P_{E V}$ is defined as:

$$
P_{E V}(t)=u_{E V}(t) \cdot i_{c h}
$$

\subsection{Simulation results}

The probabilistic load curve simulations were carried out for UFCS utilisation scenarios of $50 \mathrm{EV} /$ day, $100 \mathrm{EV} /$ day and 200 EV/day with input data distribution as explained in Fig. 2 to Fig. 4 . The objective charging time was taken $t_{c h}=5 \mathrm{~min}$ from $\operatorname{SoC}(\mathrm{t})=S_{0} C_{i} \ldots 100 \%$ for each EV. The charger efficiency was taken $\eta_{c o n v}=95 \%$. For each utilisation scenario, 10'000 Monte Carlo iterations were made based on other authors' experience [4] [5].

The simulated charging power values are shown in Table 2 , where the median value represents the lower $50 \%$ of all the iterations and the $3^{\text {rd }}$ quartile the lower $75 \%$, respectively as known from the descriptive statistics theory.

\begin{tabular}{|l|l|l|l|}
\hline EV/day & Station max $[\mathrm{kW}]$ & \multicolumn{3}{|l|}{ Per single EV [kW] } \\
\hline 50 & $1 ' 421$ & Median: & 214 \\
\hline 100 & $1^{\prime} 733$ & $3^{\text {rd }}$ quartile: & 284 \\
\hline 200 & $2 ' 218$ & Max: & 697 \\
\hline
\end{tabular}

Table 2: Power ratings of an UFCS for $t_{c h}=5 \mathrm{~min}$

The energies transferred from the utility grid to the vehicles during a day are shown in Table 3.

\begin{tabular}{|l|l|l|l|}
\hline EV/day & Median & $3^{\text {rd }}$ quartile & Max \\
\hline 50 & $907 \mathrm{~kW} \cdot \mathrm{h}$ & $942 \mathrm{~kW} \cdot \mathrm{h}$ & $1^{\prime} 113 \mathrm{~kW} \cdot \mathrm{h}$ \\
\hline 100 & $1^{\prime} 851 \mathrm{~kW} \cdot \mathrm{h}$ & $1^{\prime} 901 \mathrm{~kW} \cdot \mathrm{h}$ & $2^{\prime} 220 \mathrm{~kW} \cdot \mathrm{h}$ \\
\hline 200 & $3^{\prime} 652 \mathrm{~kW} \cdot \mathrm{h}$ & $3^{\prime} 729 \mathrm{~kW} \cdot \mathrm{h}$ & $4^{\prime} 061 \mathrm{~kW} \cdot \mathrm{h}$ \\
\hline
\end{tabular}

Table 3: Energy ratings for and UFCS for $t_{c h}=5 \mathrm{~min}$

If the vehicles arrive at an UFCS with an interval shorter than charging time, the grid burden is summed up of individual charging curves (Fig. 5). In implementation, to enable simultaneous charging of multiple vehicles, corresponding number of $\mathrm{EV}$ interfaces, i.e. charging ports must be foreseen.

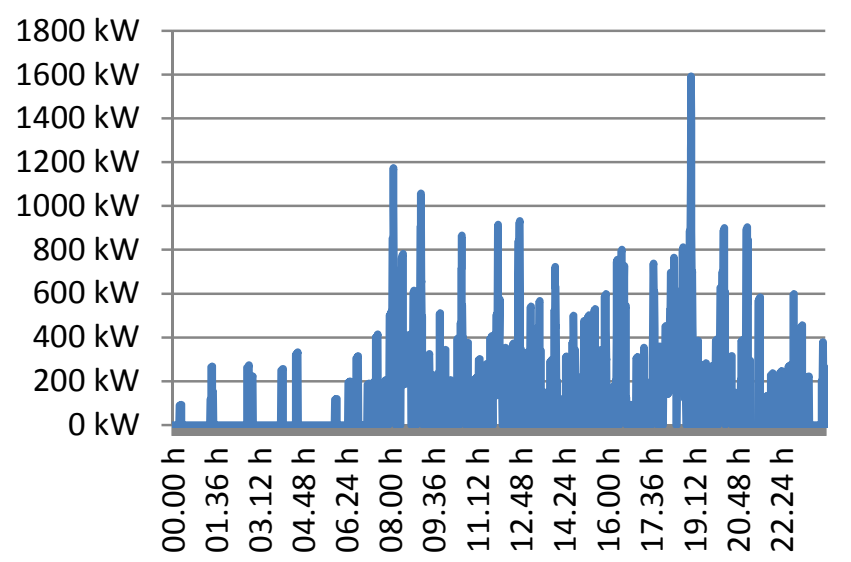

Fig. 5: A sample unmanaged grid load curve for $200 \mathrm{EV} /$ day

\section{Load management and buffering}

\subsection{Scheduling with power limitation}

For economic reasons, the design of an UFCS should be based rather on optimal than on worst-case values. As for charging power limitation, the $3^{\text {rd }}$ quartile values permit to recharge an EV within 5 minutes at $75 \%$ of all cases (Table $2, P_{c h}=284 \mathrm{~kW}$ ). The same can be said about the number of charging ports per UFCS. However, with limited charging power and EV connection ports, waiting queues emerge (Table 4) [6]. A sample load diagram with scheduling and charging power limitation is depicted in Fig. 6.

\begin{tabular}{|l|l|l|l|l|}
\hline \multirow{2}{*}{$\begin{array}{l}\text { EV/ } \\
\text { day }\end{array}$} & \multicolumn{2}{|l|}{$\mathrm{N}^{\circ}$ of EV ports } & \multicolumn{2}{|c|}{ Waiting time [min] } \\
\cline { 2 - 5 } & Simulations'max & Proposed & $3^{\text {rd }}$ quartile & Max \\
\hline 50 & 4 & 1 & 5.6 & 21.9 \\
\hline 100 & 6 & 2 & 2.5 & 11.3 \\
\hline 200 & 8 & 3 & 1.0 & 10.4 \\
\hline
\end{tabular}

Table 4: Queues at charging power limited to $3^{\text {rd }}$ quartile

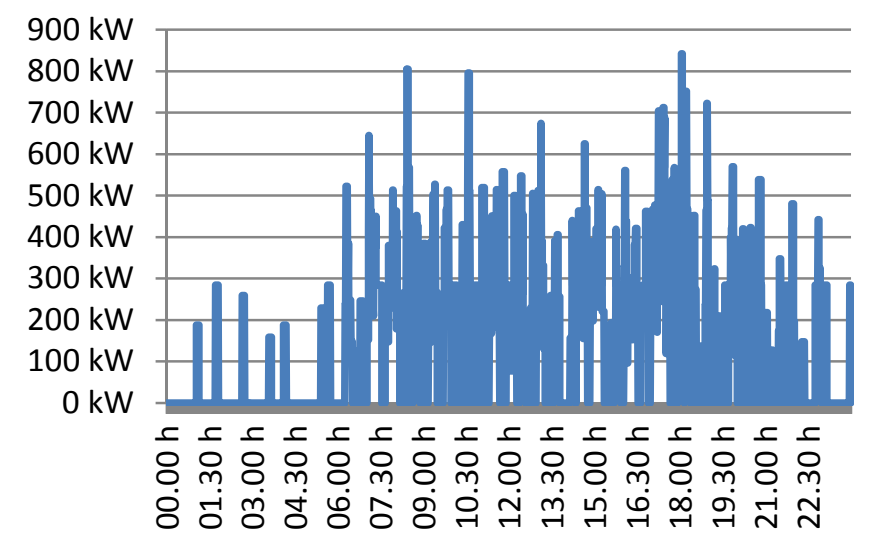

Fig. 6: A sample scheduled load curve for $200 \mathrm{EV} /$ day

With the power limitation related scheduling, the maximum grid burden of an UFCS can be reduced to a fixed value, defined by the number of EV ports and charging power allocated to each port.

\subsection{The concept of buffering}

Even with scheduling, the UFCS load on a utility grid has a highly fluctuating character. Such short-time peaks necessitate overdimensioning of the infrastructure, including cables, transformers and switchgear. A possibility of peak mitigation lies in partial decoupling of the load from the grid, which is done by energy storage elements acting as an intermediate buffer between the EV and the utility grid. The same approach has already been applied in fast refilling of compressed air powered vehicles [7]. The major components of a buffered UFCS are (Fig. 7):

1. Low power charger LPC keeps the SoC to guarantee EV charging power availability and limits the grid load to an average value. It must transmit only charging power for the stationary buffer. During EV charging, it operates in parallel with the intermediate buffer IB. 
2. High power charger HPC charges the EV, drawing energy both from the grid and the buffer. It must transmit the full EV charging power.

3. The intermediate buffer IB, connected to the dc bus between the LPC and HPC, charges with lower power from the grid and discharges into the vehicle(s).

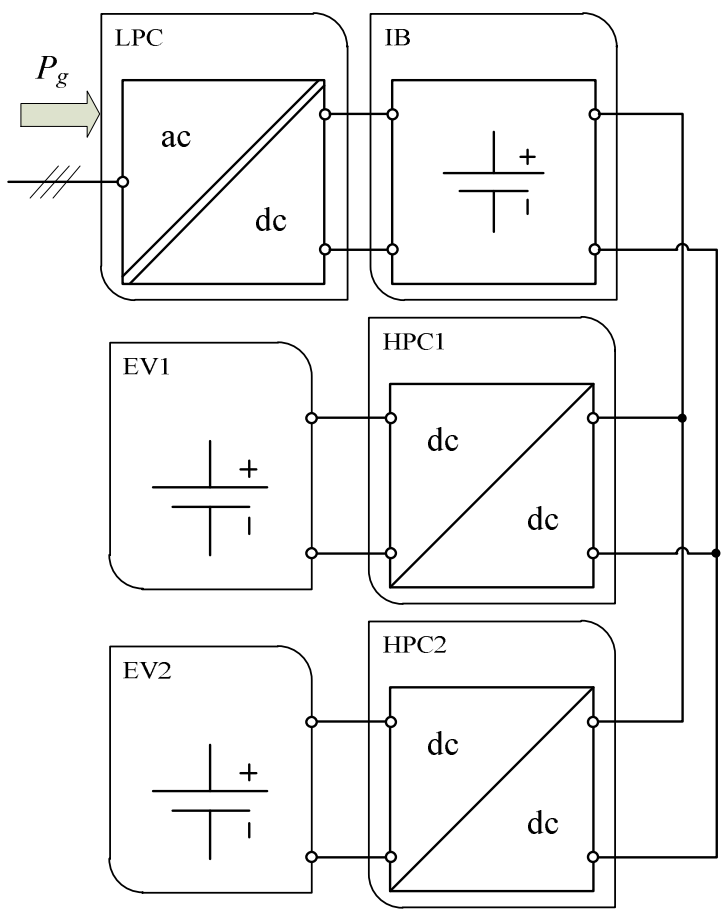

Fig. 7: A buffered UFCS with two EV ports

The buffered UFCS design must take into account the system's overall energy balance over specified time:

$$
\int_{0}^{t_{1}} P_{g}(t) \cdot d t=\sum_{i=1}^{n} \int_{0}^{t_{1}} P_{H P C, i}(t) \cdot d t+\int_{0}^{t_{1}} P_{I B}(t) \cdot d t
$$

The cumulative buffered power, i.e. energy is expressed as:

$$
E_{I B}(t)=\int_{0}^{t} P_{I B}(\tau) \cdot d \tau
$$

The necessary capacity for the intermediate buffer can be expressed as:

$$
E_{I B}=\max _{0 \ldots 24 h} E_{I B}(t)-\min _{0 \ldots 24 h} E_{I B}(t)
$$

While the intermediate buffer charging power is limited by the available grid power $P_{g, \max }$, the discharging power is defined by the difference between the grid and EV charging power. In an ultimate case the EV charging power is provided only by the buffer, like by following the real-time tariff. Thus,

$$
P_{I B}=-n_{E V} \cdot P_{L P C}
$$

The minus sign “_“ in Equation (10) means that the buffer acts as a source, while during buffering from the utility grid it acts as a sink.

\subsection{Example of power levelling}

The levelling means suppressing load variations seen from the grid side; this is done by setting the objective grid power $P_{g, o b j}(t)$ to follow load moving average value over a predefined period $T$ rather than its instantaneous value:

$$
P_{g, o b j}(t) \approx \frac{1}{T} \cdot \int_{t-T}^{t} \sum_{i=1}^{n} P_{H P C, i} \cdot d \tau
$$

It must be commented, that in Equation (11), the energy is assumed to be stored and converted without losses. In example (Table 5), the averaging period $T=1 \mathrm{~h}$ and already scheduled charging power as described in Table 4.

\begin{tabular}{|l|l|l|l|}
\hline $\begin{array}{l}\text { EV/ } \\
\text { day }\end{array}$ & $\begin{array}{l}\text { Buffered } \\
\text { power }[\mathrm{kW}]\end{array}$ & $\begin{array}{l}\text { Buffered } \\
\text { energy }[\mathrm{kW} \cdot \mathrm{h}]\end{array}$ & $\begin{array}{l}\text { Grid connection } \\
{[\mathrm{kW}]}\end{array}$ \\
\hline 50 & 284 & 144 & 112 \\
\hline 100 & 568 & 218 & 196 \\
\hline 200 & 852 & 334 & 426 \\
\hline
\end{tabular}

Table 5: Values for a buffered UFCS with levelling strategy

For an example of $200 \mathrm{EV} / \mathrm{day}$, the grid connection can be downscaled approximately 5 times in comparison with unmanaged supply (Table 2) and 2 times as compared with non-buffered scheduling (Table 4). A graphical representation of levelled grid load curve is shown in Fig. 8 (to be compared with Fig. 6).

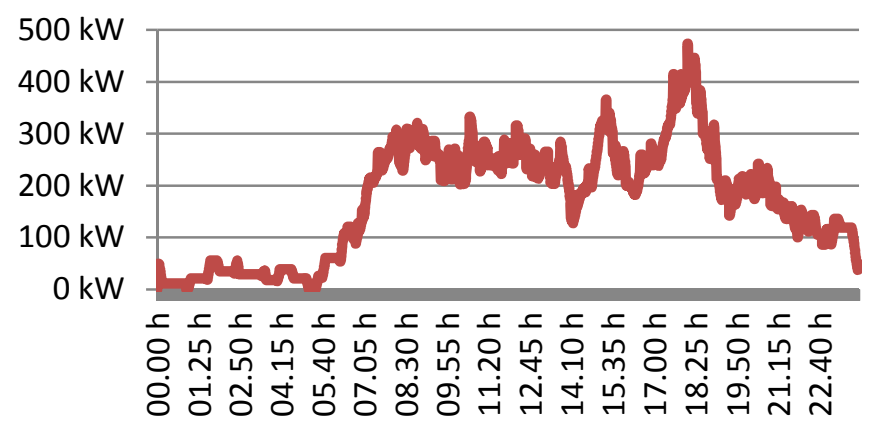

Fig. 8: A sample levelled grid load curve for $200 \mathrm{EV} /$ day

\section{Energy storage selection and interfacing}

\subsection{Qualifying criteria}

The first step in selection is to determine the buffer discharge times, expressed as the ratio between $E_{I B}$ and $P_{I B}$. From Table 5 , the discharge times vary from $23 \mathrm{~min}$ to $30 \mathrm{~min}$, falling into the grey zone between short- and long-term storages [8].

Secondly, the number of full recharge/discharge cycles helps to determine the prospective lifetime of the installed buffer. The number of daily cycles can be achieved whilst dividing the transferred energy in 24 hours (Table 3) with buffer capacity (Table 5).

Finally, the specific energy and specific power values help to determine the resulting mass and volume of the prospective intermediate buffer (Table 6). 


\begin{tabular}{|l|l|l|l|}
\hline Storage medium & $\begin{array}{l}\text { Mass } \\
{[\mathrm{t}]}\end{array}$ & $\begin{array}{l}\text { Volume } \\
{\left[\mathrm{m}^{3}\right]}\end{array}$ & $\begin{array}{l}\text { Prospective } \\
\text { lifetime }\end{array}$ \\
\hline Lithium ion battery & 3 & 1 & 1 year \\
\hline Lead-acid battery & 65 & 17 & 3 months \\
\hline Flywheel & 11 & 16 & 20 years \\
\hline Ultracapacitor & 55 & 74 & 20 years \\
\hline
\end{tabular}

Table 6: Storage media comparison for $200 \mathrm{EV} /$ day

The state of art of energy storage does not give a clear answer to the optimal storage medium selection in terms of installation space, lifetime and costs. To improve lifetime, the electrochemical batteries must be overdimensioned to narrow the SoC window. Another possibility lies in the use of the socalled second-life batteries dismantled from the vehicles or other applications and still capable to serve in an UFCS with de-rated capabilities [9].

\subsection{Storage media interfacing}

In many cases the storage medium is not connected directly to the common power bus between the primary supply and the load, but by means of a power interface either to convert the energy from one form to another or to match the voltages between the storage medium terminals and the power bus. In an intermediate buffer as shown in Fig. 7, the storage medium can be interfaced to the power bus in following ways:

1. Direct interfacing (Fig. 9a) is possible when the HPC input voltage $u_{H P C}$ tolerance is sufficiently high to accept the whole storage medium SoC-related terminal voltage $u_{\text {bat }}$ window. The energy exchange with storage medium is controlled only by the LPC and HPC.

2. Parallel active interfacing (Fig. 9b) gives an additional energy flow control degree of freedom from and to the storage medium. The HPC input voltage can be kept constant independently of the buffer's SoC.

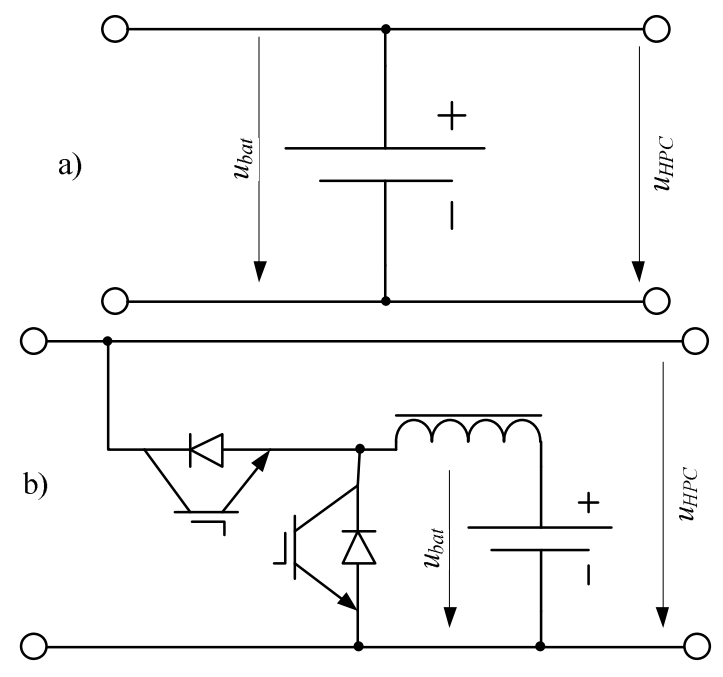

Fig. 9: Storage medium interfacing in intermediate buffer

An interface in an intermediate buffer adds complexity to the system, so direct connection should be preferred provided the HPC remains operational in whole storage medium voltage window $u_{b a t}$, like explained in Table 7.

\begin{tabular}{|l|l|}
\hline Storage medium & Voltage window \\
\hline Lead-acid & $(0.85 \ldots 1.20) \cdot u_{b a t, n}$ \\
\hline Lithium-manganese & $(0.73 \ldots 1.11) \cdot u_{b a t, n}$ \\
\hline Lithium-iron-phosphate & $(0.88 \ldots 1.25) \cdot u_{b a t, n}$ \\
\hline Lithium-titanate & $(0.67 \ldots 1.24) \cdot u_{b a t, n}$ \\
\hline Ultracapacitor & $(0.5 \ldots 1.0) \cdot u_{b a t, n}$ \\
\hline
\end{tabular}

Table 7: Operational voltage windows for storage media

\section{Connection to medium voltage utility grid}

Even with levelling, the UFCS necessitate a strong grid connection. In low voltage (LV) networks, power levels such as in Table 5 require cables with large cross-section and vicinity of a transformer substation. So it might be argued, that any UFCS is connected to a medium voltage (MV) grid over an interface.

A conventional method of interfacing an UFCS to MV grid would be that through an isolated low frequency power transformer (Fig. 10) [5]. As the low frequency passive components add weight and volume to the system, alternative solutions based on ac/dc conversion on the MV side should be considered with medium frequency (MF) galvanic isolation.

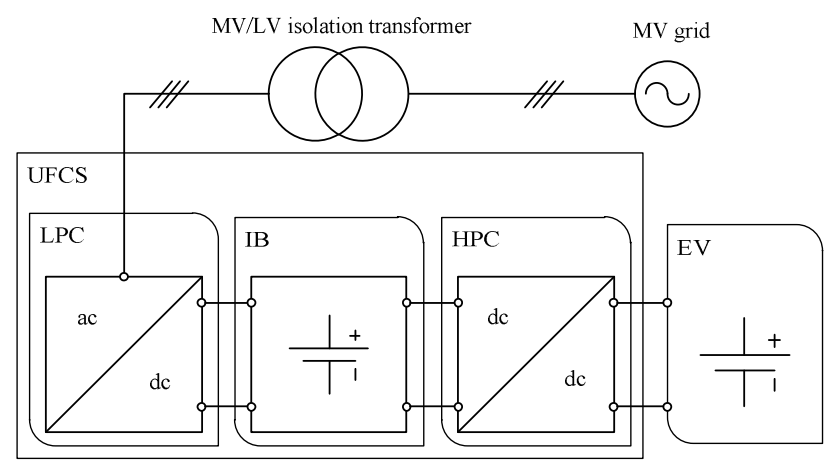

Fig. 10: Conventional UFCS MV connection

The proposed UFCS architecture is based on the cascaded Hbridge converter topology with split integrated storage (CHB+SIS, Fig. 11) [10]. Besides CHB topology and integrated storage, one of the distinctive characteristics of this design is the paralleled high power charger (PHPC).

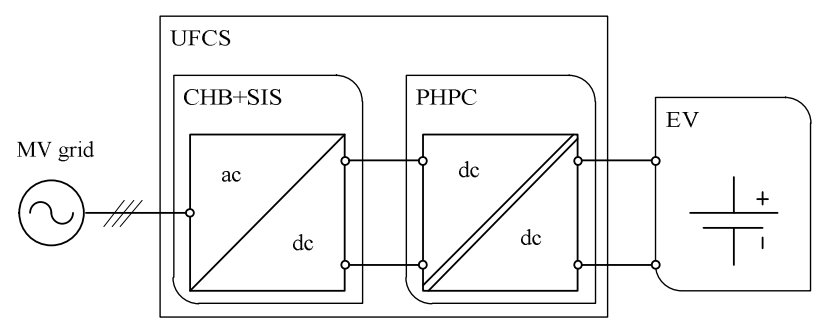

Fig. 11: CHB-based UFCS

A diagram of CHB-based UFCS is shown in Fig. 12. The proposed structure is bidirectional in terms of power flow and can also provide reactive compensation and frequency control. Besides the absence of bulky low frequency power transformer, the $\mathrm{CHB}$ design offers lower harmonic distortion, better fault handling and control flexibility. 


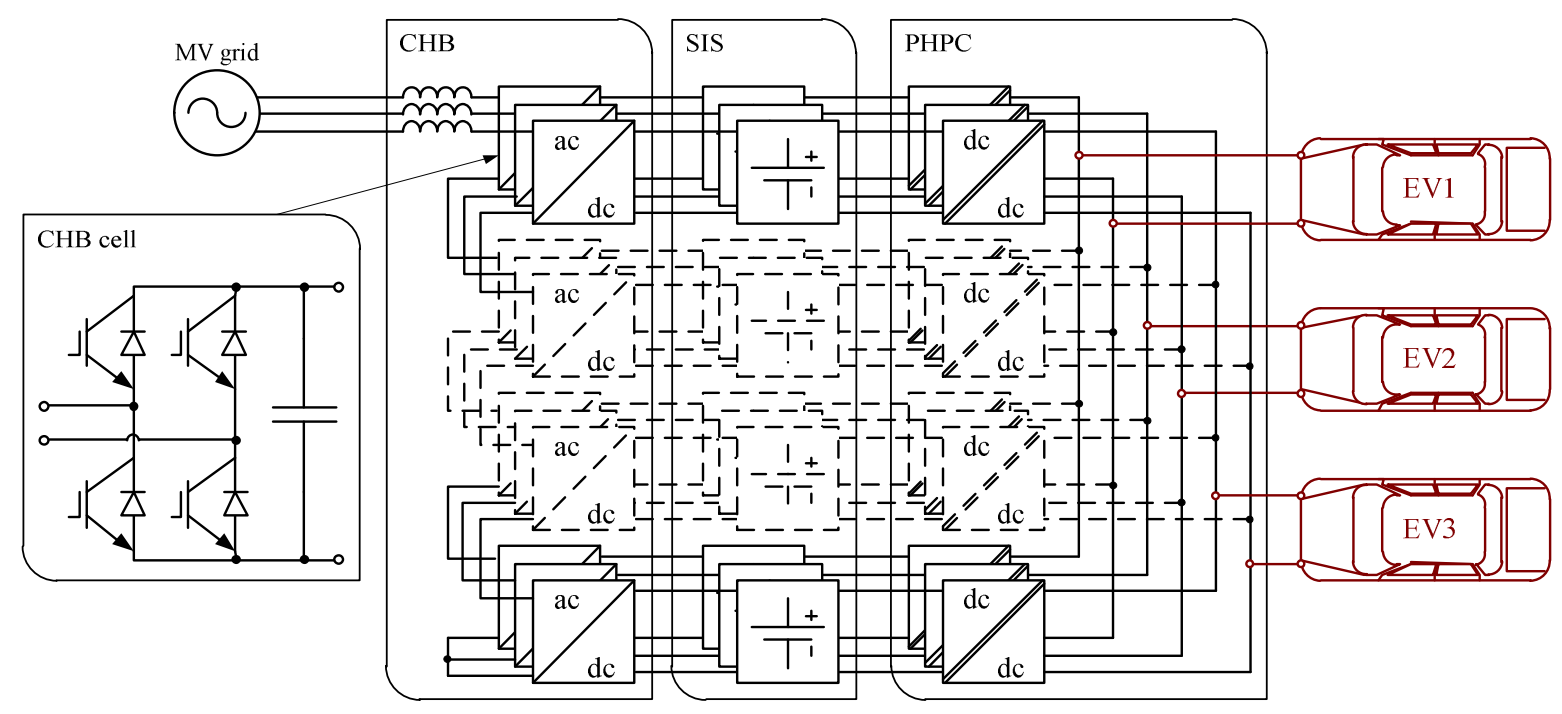

Fig. 12: Architecture of an UFCS based on the cascaded H-bridge and split integrated storage

\section{Conclusions}

The research activities summarised in current paper allow drawing the following main conclusions:

1. Ultrafast charging impact on the utility grid grows with the EV market penetration. During peak hours, the charging powers of single EVs sum up.

2. The peaks on the grid side might be alleviated with load scheduling, with the trade-off of waiting queues and longer charging times thanks to power limitation.

3. The load levelling strategy by the application of intermediate buffering helps furthermore to downsize the necessary grid connection. Levelling is more effective at low UFCS utilisation, when the moving average charging power exceeds the charging power of a single EV.

4. There are presently no optimal storage solution in terms of weight, volume, lifetime and cost. A possibility lies in re-using the de-rated "second-life" batteries.

5. A promising UFCS architecture is CHB+SIS based, offering more compactness, fault handling features and control flexibility than power transformer based one.

As the next step, the project team is designing an ultrafast charging demonstrator, capable of recharging a stock EV from the 32 A household socket during 5 minutes.

\section{Acknowledgements}

The authors would like to thank the Competence Centre Energy and Mobility (CCEM) as well as the Swisselectric Research for their continuous support to the EPFL industrial electronics laboratory research team in the field of EV infrastructure related studies.

\section{References}

[1] M. Kamachi, H. Miyamoto, Y. Sano. "Development of Power Management System for Electric Vehicle "i-
MiEV"”, in the Proc. of International Power Electronics Conference, pp. 2949-2955 (2010).

[2] H.M. Magraner. "Ultra-Fast DC Charging Stations", ECPE Workshop on Power Electronics for Electric Vehicles, 23 p. (2011).

[3] GS Yuasa product review 2011 [Online]. Available: http://www.gsyuasa-lp.com/Products.html [Accessed December 11, 2011].

[4] K. Yunus, H. Zelaya de la Parra, M. Reza. "Distribution Grid Impact of Plug-In Electric Vehicles Charging at Fast Charging Stations Using Stochastic Charging Model" in the Proc. of European Power Electronics Conference, 11 p. (2011).

[5] S. Bai., Y. Du., S. Lukic. "Optimum design of an EV/PHEV charging station with DC bus and storage system", in the Proc. of IEEE Energy Conversion Conference and Exposition, pp. 1178-1184 (2010).

[6] H. Hõimoja, A. Rufer. "Infrastructure Issues Regarding the Ultrafast Charging of Electric Vehicles", International Advanced Mobility Forum, 8 p. (2012).

[7] A. Rufer, S. Lemofouet, M. Habisreutinger, M. Heidari, A. Leuba. "Driving and Filling Personal Vehicles - The Questions of Energy - and Power - Density", in Geneva World Engineers' Convention, 9 p. (2011).

[8] M.A. Guerrero, E. Romero, F. Barrero. "Overview of Medium Scale Energy Storage Systems" in the Proc. of Compatibility and Power Electronics, pp. 93-100 (2009).

[9] H. Hõimoja, M. Vasiladiotis, S. Grioni, M. Capezzali, A. Rufer, H. B. Püttgen. "Toward Ultrafast Charging Solutions of Electric Vehicles", Cigré 2012 Paris session, 8 p. (2012).

[10]L. Maharjan, S. Inoue, H. Akagi, J. Asakura. "A transformerless battery energy storage system based on a multilevel cascade PWM converter", in the Proc. of IEEE Power Electronics Specialists Conference, pp. 47984804 (2008). 